\title{
Benign Extrahepatic Bile Duct Neoplasm
}

National Cancer Institute

\section{Source}

National Cancer Institute. Benign Extrahepatic Bile Duct Neoplasm. NCI Thesaurus. Code C4776.

A non-metastasizing neoplasm that arises from the extrahepatic bile ducts. 